\title{
Asymptotics of Determinants and Traces of Toeplitz Matrices with Symbols in Weighted Wiener Algebras
}

\author{
Alexei Yu. Karlovich
}

\begin{abstract}
We prove asymptotic formulas for determinants and traces of finite block Toeplitz matrices with symbols belonging to Wiener algebras with weights satisfying natural submultiplicativity, monotonicity, and regularity conditions. The remainders in these formulas depend on the weights and go rapidly to zero for very smooth symbols. These formulas refine or extend some previous results by Szegó, Widom, Böttcher, and Silbermann.
\end{abstract}

Keywords. Block Toeplitz matrix, determinant, trace, weighted Wiener algebra, canonical Wiener-Hopf factorization, strong Szegö-Widom limit theorem.

Mathematics Subject Classification (2000). Primary 47B35, secondary 15A15, 47B10, 47L20.

\section{Introduction and main results}

1.1. Finite block Toeplitz matrices. Let $\mathbb{Z}, \mathbb{N}, \mathbb{Z}_{+}$, and $\mathbb{C}$ be the sets of integers, positive integers, nonnegative integers, and all complex numbers, respectively. Suppose $N \in \mathbb{N}$. For a Banach space $X$, let $X_{N}$ and $X_{N \times N}$ be the spaces of vectors and matrices with entries in $X$. Let $\mathbb{T}$ be the unit circle. For $1 \leq p \leq \infty$, let $L^{p}:=L^{p}(\mathbb{T})$ and $H^{p}:=H^{p}(\mathbb{T})$ be the standard Lebesgue and Hardy spaces of the unit circle. For $a \in L_{N \times N}^{1}$ one can define

$$
a_{k}=\frac{1}{2 \pi} \int_{0}^{2 \pi} a\left(e^{i \theta}\right) e^{-i k \theta} d \theta \quad(k \in \mathbb{Z}),
$$

the sequence of the Fourier coefficients of $a$. Let $I$ be the identity operator, $P$ be the Riesz projection of $L^{2}$ onto $H^{2}, Q:=I-P$, and define $I, P$, and $Q$ on $L_{N}^{2}$ elementwise. For $a \in L_{N \times N}^{\infty}$ and $t \in \mathbb{T}$, put $\widetilde{a}(t):=a\left(\frac{1}{t}\right)$ and $(J a)(t):=t^{-1} \widetilde{a}(t)$.

A. Yu. Karlovich: Universidade do Minho, Escola de Ciências, Centro de Matemática, Campus de Gualtar, 4710-057, Braga, Portugal; oleksiy@math.uminho.pt Current address: Departamento de Matemática, Instituto Superior Técnico, Av. Rovisco Pais 1, 1049-001, Lisbon, Portugal; akarlov@math.ist.utl.pt 
Define Toeplitz operators

$$
T(a):=P a P|\operatorname{Im} P, \quad T(\widetilde{a}):=J Q a Q J| \operatorname{Im} P
$$

and Hankel operators

$$
H(a):=P a Q J|\operatorname{Im} P, \quad H(\widetilde{a}):=J Q a P| \operatorname{Im} P .
$$

The function $a$ is called the symbol of $T(a), T(\widetilde{a}), H(a), H(\widetilde{a})$. We are interested in the asymptotic behavior of the determinants and traces of finite block Toeplitz matrices $T_{n}(a)=\left[a_{j-k}\right]_{j, k=0}^{n}$ generated by (the Fourier coefficients of) the symbol $a$ as $n \rightarrow \infty$. Many results in this direction are contained in the books by Grenander and Szegő [9], Böttcher and Silbermann [2, 3, 4], and Simon [14].

1.2. Szegö-Widom limit theorems. Let us formulate precisely the most relevant results. Let $K_{N \times N}^{2}$ be the Krein algebra [11] of matrix functions $a$ in $L_{N \times N}^{\infty}$ satisfying

$$
\sum_{k=-\infty}^{\infty}\left\|a_{k}\right\|^{2}(|k|+1)<\infty
$$

where $\|\cdot\|$ is any matrix norm on $\mathbb{C}_{N \times N}$. The following beautiful theorem about the asymptotics of finite block Toeplitz matrices was proved by Widom [16].

Theorem 1.1 (see [16, Theorem 6.1]). Let $N \geq 1$. If $a \in K_{N \times N}^{2}$ and the Toeplitz operators $T(a)$ and $T(\widetilde{a})$ are invertible on $H_{N}^{2}$, then $T(a) T\left(a^{-1}\right)-I$ is of trace class and, with appropriate branches of the logarithm,

$$
\log \operatorname{det} T_{n}(a)=(n+1) \log G(a)+\log \operatorname{det} T(a) T\left(a^{-1}\right)+o(1) \quad \text { as } n \rightarrow \infty,
$$

where

$$
G(a):=\lim _{r \rightarrow 1-0} \exp \left(\frac{1}{2 \pi} \int_{0}^{2 \pi} \log \operatorname{det} a_{r}\left(e^{i \theta}\right) d \theta\right), \quad a_{r}\left(e^{i \theta}\right):=\sum_{n=-\infty}^{\infty} a_{n} r^{|n|} e^{i n \theta} .
$$

Here $\operatorname{det} T(a) T\left(a^{-1}\right)$ refers to the determinant defined for operators on Hilbert space differing from the identity by an operator of trace class [8, Ch. 4].

The proof of the above result in a more general form is contained in $[2$, Theorem 6.11] and [4, Theorem 10.30] (in this connection see also [5]).

Let $\lambda_{1}^{(n)}, \ldots, \lambda_{(n+1) N}^{(n)}$ denote the eigenvalues of $T_{n}(a)$ repeated according to their algebraic multiplicity. Let $\operatorname{sp} A$ denote the spectrum of a bounded linear operator $A$ and $\operatorname{tr} M$ denote the trace of a matrix $M$. Theorem 1.1 is equivalent to the assertion

$$
\sum_{i} \log \lambda_{i}^{(n)}=\operatorname{tr} \log T_{n}(a)=(n+1) \log G(a)+\log \operatorname{det} T(a) T\left(a^{-1}\right)+o(1) .
$$


Widom [16] noticed that Theorem 1.1 yields even a description of the asymptotic behavior of $\operatorname{tr} f\left(T_{n}(a)\right)$ if one replaces $f(\lambda)=\log \lambda$ by an arbitrary function $f$ analytic in an open neighborhood of the union $\operatorname{sp} T(a) \cup \operatorname{sp} T(\widetilde{a})$ (we henceforth call such $f$ simply analytic on $\operatorname{sp} T(a) \cup \operatorname{sp} T(\widetilde{a}))$.

Theorem 1.2 (see [16, Theorem 6.2]). Let $N \geq 1$. If $a \in K_{N \times N}^{2}$ and if $f$ is analytic on $\operatorname{sp} T(a) \cup \operatorname{sp} T(\widetilde{a})$, then

$$
\operatorname{tr} f\left(T_{n}(a)\right)=(n+1) G_{f}(a)+E_{f}(a)+o(1) \text { as } n \rightarrow \infty,
$$

where

$$
\begin{aligned}
G_{f}(a) & :=\frac{1}{2 \pi} \int_{0}^{2 \pi}(\operatorname{tr} f(a))\left(e^{i \theta}\right) d \theta \\
E_{f}(a) & :=\frac{1}{2 \pi i} \int_{\partial \Omega} f(\lambda) \frac{d}{d \lambda} \log \operatorname{det} T[a-\lambda] T\left[(a-\lambda)^{-1}\right] d \lambda,
\end{aligned}
$$

and $\Omega$ is any bounded open set containing $\operatorname{sp} T(a) \cup \operatorname{sp} T(\widetilde{a})$ on the closure of which $f$ is analytic.

The proof of Theorem 1.2 for smooth symbols $a$ is also given in [4, Section 10.90].

In the scalar case $(N=1)$ Theorems 1.1 and 1.2 go back to Gabor Szegő (see [9] and historical remarks in $[2,3,4,14]$ ).

1.3. The Böttcher-Silbermann higher order asymptotic formulas. Following [16] and [4, Sections 7.5-7.6], for $n \in \mathbb{Z}_{+}$and $a \in L_{N \times N}^{\infty}$ define the operators $P_{n}$ and $Q_{n}$ on $H_{N}^{2}$ by

$$
P_{n}: \sum_{k=0}^{\infty} a_{k} t^{k} \mapsto \sum_{k=0}^{n} a_{k} t^{k}, \quad Q_{n}:=I-P_{n} .
$$

The operator $P_{n} T(a) P_{n}: P_{n} H_{N}^{2} \rightarrow P_{n} H_{N}^{2}$ may be identified with the finite block Toeplitz matrix $T_{n}(a):=\left[a_{j-k}\right]_{j, k=0}^{n}$. For a unital Banach algebra $A$ we will denote by $G A$ the group of all invertible elements of $A$. Put

$$
H_{ \pm}^{\infty}:=\left\{a \in L^{\infty}: a_{\mp n}=0 \text { for } n \in \mathbb{N}\right\}
$$

and for a Banach subalgebra $\mathcal{A}$ of $L^{\infty}$, put $\mathcal{A}_{N \times N}^{ \pm}:=\left(\mathcal{A} \cap H_{ \pm}^{\infty}\right)_{N \times N}$. One says that $a \in \mathcal{A}_{N \times N}$ admits canonical right and left Wiener-Hopf (WH) factorizations in $\mathcal{A}_{N \times N}$ if there are functions $u_{+}, v_{+} \in G \mathcal{A}_{N \times N}^{+}$and $u_{-}, v_{-} \in G \mathcal{A}_{N \times N}^{-}$ such that $a=u_{-} u_{+}=v_{+} v_{-}$.

If $a$ is smooth enough one can expect a higher speed of convergence in (1) and (3). Let $\omega: \mathbb{Z} \rightarrow(0, \infty)$ be a weight. Consider weighted Wiener algebras

$$
\left(W_{\omega}\right)_{N \times N}:=\left\{a: \mathbb{T} \rightarrow \mathbb{C}_{N \times N}: a(t)=\sum_{n=-\infty}^{\infty} a_{n} t^{n}, \sum_{n=-\infty}^{\infty}\left\|a_{n}\right\| \omega(n)<\infty\right\} .
$$


If $\omega: \mathbb{Z} \rightarrow[1, \infty)$ is a power weight of the form

$$
\omega(n):= \begin{cases}(-n+1)^{\alpha} & \text { for } n \in \mathbb{Z} \backslash \mathbb{Z}_{+}, \quad(\alpha, \beta>0), \\ (n+1)^{\beta} & \text { for } n \in \mathbb{Z}_{+},\end{cases}
$$

then $\left(W_{\omega}\right)_{N \times N}$ will be denoted by $W_{N \times N}^{\alpha, \beta}$. Böttcher and Silbermann [1] proved among other things the following result.

Theorem 1.3. Let $N \geq 1$. Suppose $a \in W_{N \times N}^{\alpha, \beta}(\alpha, \beta>0)$ and the Toeplitz operators $T(a)$ and $T(\widetilde{a})$ are invertible on $H_{N}^{2}$.

(a) The matrix function a admits canonical right and left Wiener-Hopf factorizations $a=u_{-} u_{+}=v_{+} v_{-}$in $W_{N \times N}^{\alpha, \beta}$.

(b) If $\alpha+\beta>1$, then $T(a) T\left(a^{-1}\right)-I$ is of trace class and (1) is true with $o$ (1) replaced by $o\left(1 / n^{\alpha+\beta-1}\right)$.

(c) If $\alpha+\beta>\frac{1}{p}$ for some $p \in \mathbb{N} \backslash\{1\}$, then there exist a constant $\widetilde{E}(a) \neq 0$ such that

$$
\begin{aligned}
\log \operatorname{det} T_{n}(a)= & (n+1) \log G(a)+\log \widetilde{E}(a) \\
& +\operatorname{tr}\left[\sum_{\ell=1}^{n} \sum_{j=1}^{p-1} \frac{1}{j}\left(\sum_{k=0}^{p-j-1} G_{\ell, k}(b, c)\right)^{j}\right]+o\left(1 / n^{(\alpha+\beta) p-1}\right)
\end{aligned}
$$

as $n \rightarrow \infty$, where the correcting terms $G_{\ell, k}(b, c)$ are given by

$$
G_{\ell, k}(b, c):=P_{0} T(c) Q_{\ell}\left(Q_{\ell} H(b) H(\widetilde{c}) Q_{\ell}\right)^{k} Q_{\ell} T(b) P_{0} \quad\left(\ell, k \in \mathbb{Z}_{+}\right)
$$

and the functions $b, c$ are given $b y b:=v_{-} u_{+}^{-1}$ and $c:=u_{-}^{-1} v_{+}$.

The proof of Theorem 1.3 (for $p=1,2,3$ ) is contained in [2, Sections 6.186.20] and in [4, Theorem 10.35 and Corollary 10.38].

1.4. Our main results. The aim of this paper is to extend or refine the above results in the case of symbols that belong to more general weighted Wiener algebras $\left(W_{\omega}\right)_{N \times N}$, where the weight $\omega: \mathbb{Z} \rightarrow[1, \infty)$ satisfies

$$
\begin{aligned}
& 1 \leq \omega(i+j) \leq \omega(i) \omega(j) \quad(i, j \in \mathbb{Z}) \\
& \omega( \pm n) \leq \omega( \pm(n+1)) \quad\left(n \in \mathbb{Z}_{+}\right) \\
& \lim _{n \rightarrow+\infty} \sqrt[n]{\omega(n)}=\lim _{n \rightarrow+\infty} \frac{1}{\sqrt[n]{\omega(-n)}}=1 .
\end{aligned}
$$

For $n \in \mathbb{Z}_{+}$, put

$$
\varphi_{n}:=[\omega(n+1) \omega(-(n+1))]^{-1} .
$$

Our first main result is the following extension of Theorem 1.3. 
Theorem 1.4. Let $N \geq 1$ and let $\omega: \mathbb{Z} \rightarrow[1, \infty)$ be a weight satisfying $(7)-(9)$. Suppose $\Sigma$ is a compact set in the complex plane, $a: \Sigma \rightarrow\left(W_{\omega}\right)_{N \times N}$ is a continuous function, and the Toeplitz operators $T(a(\lambda))$ and $T\left([a(\lambda)]^{\sim}\right)$ are invertible on $H_{N}^{2}$ for all $\lambda \in \Sigma$.

(a) For every $\lambda \in \Sigma$, the function $a(\lambda): \mathbb{T} \rightarrow \mathbb{C}$ admits canonical right and left Wiener-Hopf factorizations $a(\lambda)=u_{-}(\lambda) u_{+}(\lambda)=v_{+}(\lambda) v_{-}(\lambda)$. These factorizations can be chosen so that $b, c: \Sigma \rightarrow\left(W_{\omega}\right)_{N \times N}$ given by $b:=v_{-} u_{+}^{-1}$ and $c:=u_{-}^{-1} v_{+}$are continuous.

(b) If $\sum_{k=1}^{\infty} \varphi_{k}<\infty$, then $T(a(\lambda)) T\left([a(\lambda)]^{-1}\right)-I$ is of trace class for every $\lambda \in \Sigma$ and

$$
\begin{aligned}
\log \operatorname{det} T_{n}(a(\lambda))= & (n+1) \log G(a(\lambda)) \\
& +\log \operatorname{det} T\left(a(\lambda) T\left([a(\lambda)]^{-1}\right)+o\left(\sum_{k=n+1}^{\infty} \varphi_{k}\right)\right.
\end{aligned}
$$

as $n \rightarrow \infty$, where the convergence is uniform with respect to $\lambda \in \Sigma$.

(c) If $\sum_{k=1}^{\infty} \varphi_{k}^{p}<\infty$ for some $p \in \mathbb{N} \backslash\{1\}$, then for every $\lambda \in \Sigma$ there exists a constant $\widetilde{E}(a, \lambda) \neq 0$ such that

$$
\begin{aligned}
\log \operatorname{det} T_{n}(a(\lambda))= & (n+1) \log G(a(\lambda))+\log \widetilde{E}(a, \lambda) \\
& +\operatorname{tr}\left[\sum_{\ell=1}^{n} \sum_{j=1}^{p-1} \frac{1}{j}\left(\sum_{k=0}^{p-j-1} G_{\ell, k}(b(\lambda), c(\lambda))\right)^{j}\right] \\
& +o\left(\sum_{k=n+1}^{\infty} \varphi_{k}^{p}\right)
\end{aligned}
$$

as $n \rightarrow \infty$, where the correcting terms $G_{\ell, k}(b(\lambda), c(\lambda))$ are defined by (6) and the functions $b, c: \Sigma \rightarrow\left(W_{\omega}\right)_{N \times N}$ are chosen as in (a). The convergence in (11) is uniform with respect to $\lambda \in \Sigma$.

Clearly the power weight (4) satisfies all the conditions (7)-(9). So, Theorem 1.3 follows from Theorem 1.4. An example constructed in the proof of $[10$, Theorem 23] shows that Theorem 1.4 is stronger than Theorem 1.3.

Our second main result is the following refinement of Theorem 1.2.

Theorem 1.5. Let $N \geq 1$ and let $\omega: \mathbb{Z} \rightarrow[1, \infty)$ be a weight satisfying (7)-(9). If $a \in\left(W_{\omega}\right)_{N \times N}, f$ is analytic on $\operatorname{sp} T(a) \cup \operatorname{sp} T(\widetilde{a})$, and $\sum_{k=1}^{\infty} \varphi_{k}<\infty$, then (3) is true with o(1) replaced by the same o(...) as in (10).

1.5. On the higher order asymptotic formula of Vasil'ev, Maksimenko, and Simonenko. For Wiener algebras with power weights Theorem 1.5 has a very simple form and is obviously predicted by the Böttcher-Silbermann Theorem 1.3(b). 
Corollary 1.6. Let $N \geq 1$. Suppose $a \in W_{N \times N}^{\alpha, \beta}(\alpha, \beta>0)$ and $f$ is analytic on $\operatorname{sp} T(a) \cup \operatorname{sp} T(\widetilde{a})$. If $\alpha+\beta>1$, then (3) is true with o(1) replaced by $o\left(1 / n^{\alpha+\beta-1}\right)$.

In particular, if $\gamma>1$ and $a \in W_{N \times N}^{\gamma / 2, \gamma / 2}$, then (3) is true with $o(1)$ replaced by $o\left(1 / n^{\gamma-1}\right)$. Recently Vasil'ev, Maksimenko, and Simonenko [15] stated (without proofs) that if $a$ satisfies

$$
\sum_{k=-\infty}^{\infty}\left\|a_{k}\right\|+\sum_{k=-\infty}^{\infty}\left\|a_{k}\right\|^{2}(|k|+1)^{\gamma}<\infty
$$

for some $\gamma>1$, then the $o(1)$ in (3) can be replaced by $o\left(1 / n^{\gamma-1}\right)$. Obviously, the class $\left(W \cap F \ell_{\gamma / 2}^{2}\right)_{N \times N}$ of matrix functions a satisfying (12) is larger than $W_{N \times N}^{\gamma / 2, \gamma / 2}$ for a fixed $\gamma$. However, Theorems 1.3-1.5 allow different behavior of the negative and positive parts of the Fourier series. Consider the following example. For a given $\gamma>1$, put $\beta=\frac{1}{2}(1+\gamma), \alpha=\frac{1}{4}(1+\gamma)$, and

$$
a(t):=a_{0}+\sum_{k=1}^{\infty} k^{-\beta} t^{-k} \quad\left(t \in \mathbb{T}, a_{0} \in \mathbb{C}\right) .
$$

Then $a \in W_{1 \times 1}^{\alpha, \gamma-\alpha}$, however $a \notin\left(W \cap F \ell_{\gamma / 2}^{2}\right)_{1 \times 1}$. This means that Theorem 1.5 is applicable to $a$ and gives the speed $o\left(1 / n^{\gamma-1}\right)$, while the theorem of Vasil'ev, Maksimenko, and Simonenko is not applicable to this function.

1.6. About this paper. This paper can be considered as a continuation of [10]. It is organized as follows. In Sections 2.1-2.2 we collect necessary facts on weighted Wiener algebras and canonical Wiener-Hopf factorizations in these algebras. Sections 2.3-2.4 contain some auxiliary estimates. In Section 3.1 we state an analogue of Theorem 1.1 for weighted Wiener algebras. Section 3.2 contains a key observation, Lemma 3.2 by Böttcher and Silbermann, which allows us to get better speed than $o(1)$ in (1) and (3) if the symbol $a$ is sufficiently smooth. In Section 3.3 we prepare the proof of Theorem 1.4 and actually show that a decomposition required by the hypothesis of Lemma 3.2 can be made even uniform with respect to a parameter $\lambda \in \Sigma$, where $\Sigma$ is a compact set as in Theorem 1.4. Sections 3.4 and 3.5 are dedicated to the proofs of Theorems 1.4 and 1.5 , respectively.

\section{Auxiliary results}

2.1. On weighted Wiener algebras. It is well known that if (7) is fulfilled, then $\left(W_{\omega}\right)_{N \times N}$ is a Banach algebra with respect to the norm

$$
\|a\|_{\omega, N}:=\sum_{k=-\infty}^{\infty}\left\|a_{k}\right\| \omega(k)
$$


and $\left(W_{\omega}\right)_{N \times N} \subset W_{N \times N} \subset C_{N \times N}$. Here $W$ is the standard Wiener algebra of scalar functions with absolutely convergent Fourier series and $C=C(\mathbb{T})$ is the set of all continuous scalar functions on $\mathbb{T}$. In the scalar case $(N=1)$ the weighted Wiener algebra is commutative. The maximal ideal space of $W_{\omega}:=$ $\left(W_{\omega}\right)_{1 \times 1}$ is homeomorphic to the unit circle $\mathbb{T}$ if $(9)$ is satisfied. These results can be found in [6, Chapter III, Section 19.4] and in [7].

2.2. Two facts on canonical Wiener-Hopf factorizations. The first fact is about stability of the factors in canonical Wiener-Hopf factorizations in weighted Wiener algebras.

Theorem 2.1. Let $N \geq 1$ and let $\omega: \mathbb{Z} \rightarrow[1, \infty)$ be a weight satisfying (7). Suppose a, $c \in\left(W_{\omega}\right)_{N \times N}$ admit canonical right and left $W H$ factorizations in the algebra $\left(W_{\omega}\right)_{N \times N}$. Then for any $\varepsilon>0$ there exists a $\delta>0$ such that if $\|a-c\|_{\omega, N}<\delta$, then for every canonical right WH factorization $a=a_{-}^{(r)} a_{+}^{(r)}$ and for every canonical left WH factorization $a=a_{+}^{(l)} a_{-}^{(l)}$ one can choose a canonical right WH factorization $c=c_{-}^{(r)} c_{+}^{(r)}$ and a canonical left WH factorization $c=$ $c_{+}^{(l)} c_{-}^{(l)}$ such that

$$
\begin{gathered}
\left\|a_{ \pm}^{(r)}-c_{ \pm}^{(r)}\right\|_{\omega, N}<\varepsilon, \quad\left\|\left[a_{ \pm}^{(r)}\right]^{-1}-\left[c_{ \pm}^{(r)}\right]^{-1}\right\|_{\omega, N}<\varepsilon \\
\left\|a_{ \pm}^{(l)}-c_{ \pm}^{(l)}\right\|_{\omega, N}<\varepsilon, \quad\left\|\left[a_{ \pm}^{(l)}\right]^{-1}-\left[c_{ \pm}^{(l)}\right]^{-1}\right\|_{\omega, N}<\varepsilon
\end{gathered}
$$

This theorem follows from a more general result due to Shubin [13] on the stability of the factors in Wiener-Hopf factorizations with the same partial indices in general decomposing algebras. Its proof can be found in $[12$, Theorem 6.15].

The second fact gives some sufficient conditions for the factorability of $a$ in the algebra $\left(W_{\omega}\right)_{N \times N}$.

Proposition 2.2. (see [10, Proposition 3]). Let $N \geq 1$ and let $\omega: \mathbb{Z} \rightarrow[1, \infty)$ be a weight satisfying (7) and (9). If $a \in\left(W_{\omega}\right)_{N \times N}$ and the Toeplitz operators $T(a)$ and $T(\widetilde{a})$ are invertible on $H_{N}^{2}$, then a admits canonical right and left Wiener-Hopf factorizations in $\left(W_{\omega}\right)_{N \times N}$.

2.3. Tails of the norms of functions in weighted Wiener algebras. For $a \in\left(W_{\omega}\right)_{N \times N}$ and $n \in \mathbb{Z}_{+}$, put

$$
R_{n}^{+}(a):=\sum_{k=n+1}^{\infty}\left\|a_{k}\right\| \omega(k), \quad R_{n}^{-}(a):=\sum_{k=n+1}^{\infty}\left\|a_{-k}\right\| \omega(-k) .
$$


Proposition 2.3. Let $\omega: \mathbb{Z} \rightarrow[1, \infty)$ be a weight satisfying (7). Let $\Sigma$ be a compact set in the complex plane and $a: \Sigma \rightarrow\left(W_{\omega}\right)_{N \times N}$ be a continuous function. Then

$$
\lim _{n \rightarrow \infty} \max _{\lambda \in \Sigma} R_{n}^{+}(a(\lambda))=0, \quad \lim _{n \rightarrow \infty} \max _{\lambda \in \Sigma} R_{n}^{-}(a(\lambda))=0 .
$$

Proof. Let us prove the first equality. Assume the contrary. Then there exist a positive constant $C>0$, a number $n_{0} \in \mathbb{N}$, and a sequence $\left\{\lambda_{n}\right\}_{n=n_{0}}^{\infty}$ such that

$$
R_{n}^{+}\left(a\left(\lambda_{n}\right)\right) \geq C \text {. }
$$

Since $\left\{\lambda_{n}\right\}_{n=n_{0}}^{\infty}$ is bounded, there is a convergent subsequence $\left\{\lambda_{n_{j}}\right\}_{j=1}^{\infty}$. Let $\lambda_{0}$ be the limit of this subsequence. Clearly, $\lambda_{0} \in \Sigma$ because $\Sigma$ is closed. Since $a$ : $\Sigma \rightarrow\left(W_{\omega}\right)_{N \times N}$ is continuous, for every $\varepsilon \in(0, C)$ there exists a $\delta>0$ such that for every $\lambda^{\prime}, \lambda^{\prime \prime} \in \Sigma$ such that $\left|\lambda^{\prime}-\lambda^{\prime \prime}\right|<\delta$ one has $\left\|a\left(\lambda^{\prime}\right)-a\left(\lambda^{\prime \prime}\right)\right\|_{\omega, N}<\varepsilon$. On the other hand, for that $\delta$ there exists a number $J \in \mathbb{N}$ such that $\left|\lambda_{n_{j}}-\lambda_{0}\right|<\delta$ for all $j \geq J$. Hence, for all $j \geq J$,

$$
\begin{aligned}
\left|R_{n_{j}}^{+}\left(a\left(\lambda_{n_{j}}\right)\right)-R_{n_{j}}^{+}\left(a\left(\lambda_{0}\right)\right)\right| & \leq \sum_{k=n_{j}+1}^{\infty}\left|\left\|\left[a\left(\lambda_{n_{j}}\right)\right]_{k}\right\|-\left\|\left[a\left(\lambda_{0}\right)\right]_{k}\right\|\right| \omega(k) \\
& \leq R_{n_{j}}^{+}\left(a\left(\lambda_{n_{j}}\right)-a\left(\lambda_{0}\right)\right) \\
& \leq\left\|a\left(\lambda_{n_{j}}\right)-a\left(\lambda_{0}\right)\right\|_{\omega, N}<\varepsilon .
\end{aligned}
$$

Since $n_{j} \geq n_{0},(15)$ implies that

$$
R_{n_{j}}^{+}\left(a\left(\lambda_{n_{j}}\right)\right) \geq C \text { for all } j \geq J .
$$

On the other hand,

$$
R_{n_{j}}^{+}\left(a\left(\lambda_{0}\right)\right) \geq R_{n_{j}}^{+}\left(a\left(\lambda_{n_{j}}\right)\right)-\left|R_{n_{j}}^{+}\left(a\left(\lambda_{0}\right)\right)-R_{n_{j}}^{+}\left(a\left(\lambda_{n_{j}}\right)\right)\right| .
$$

From (16)-(18) it follows that

$$
\liminf _{j \rightarrow \infty} R_{n_{j}}^{+}\left(a\left(\lambda_{0}\right)\right) \geq C-\varepsilon>0
$$

but this contradicts the fact that $a\left(\lambda_{0}\right) \in\left(W_{\omega}\right)_{N \times N}$. Hence, the first equality in (14) is proved. The second equality in (14) can be proved by analogy.

2.4. Norms of truncations of Hankel and Toeplitz operators. The following proposition is stated in [10, Propositions 16, 17].

Proposition 2.4. Let $N \geq 1$ and $\omega: \mathbb{Z} \rightarrow[1, \infty)$ be a weight satisfying $(7)-(8)$. If $a \in\left(W_{\omega}\right)_{N \times N}$ and $n \in \mathbb{Z}_{+}$, then

$$
\begin{aligned}
\left\|Q_{n} H(a)\right\| & \leq \frac{R_{n}^{+}(a)}{\omega(n+1)}, \quad\left\|H(\widetilde{a}) Q_{n}\right\| \leq \frac{R_{n}^{-}(a)}{\omega(-(n+1))}, \\
\left\|Q_{n} T(a) P_{0}\right\| & \leq \frac{R_{n}^{+}(a)}{\omega(n+1)}, \quad\left\|P_{0} T(a) Q_{n}\right\| \leq \frac{R_{n}^{-}(a)}{\omega(-(n+1))} .
\end{aligned}
$$




\section{Asymptotic formulas}

\subsection{The Szegö-Widom limit theorem for weighted Wiener algebras.} The following version of Theorem 1.1 was established in [10, Theorem 20(a)].

Theorem 3.1. Let $N \geq 1$ and let $\omega: \mathbb{Z} \rightarrow[1, \infty)$ be a weight satisfying (7)-(9). Suppose $a \in\left(W_{\omega}\right)_{N \times N}$ and the operators $T(a)$ and $T(\widetilde{a})$ are invertible on $H_{N}^{2}$. If $\sum_{k=1}^{\infty} \varphi_{k}<\infty$, then the operator $T(a) T\left(a^{-1}\right)-I$ is of trace class and (1) holds.

Notice that the above theorem is not a corollary of Theorem 1.1.

3.2. The Böttcher-Silbermann decomposition. The following result from [2, Section 6.16], [4, Section 10.34] is the basis for our asymptotic analysis.

Lemma 3.2. Let $N \geq 1$. Suppose $a \in L_{N \times N}^{\infty}$ satisfies the following hypotheses:

(i) there are two factorizations $a=u_{-} u_{+}=v_{+} v_{-}$, where $u_{+}, v_{+}$belong to $G\left(H_{+}^{\infty}\right)_{N \times N}$ and $u_{-}, v_{-}$belong to $G\left(H_{-}^{\infty}\right)_{N \times N}$;

(ii) $u_{-} \in C_{N \times N}$ or $u_{+} \in C_{N \times N}$.

Define the functions $b, c$ by $b:=v_{-} u_{+}^{-1}, c:=u_{-}^{-1} v_{+}$and the matrices $G_{n, k}(b, c)$ by (6). Suppose for all sufficiently large $n$ (say, $n \geq N_{0}$ ) there exists a decomposition

$$
\operatorname{tr} \log \left\{I-\sum_{k=0}^{\infty} G_{n, k}(b, c)\right\}=-\operatorname{tr} H_{n}+s_{n}
$$

where $\left\{H_{n}\right\}_{n=N_{0}}^{\infty}$ is a sequence of $N \times N$ matrices and $\left\{s_{n}\right\}_{n=N_{0}}^{\infty}$ is a sequence of complex numbers. If $\sum_{n=N_{0}}^{\infty}\left|s_{n}\right|<\infty$, then there exist a constant $\widetilde{E}(a) \neq 0$, depending on $\left\{H_{n}\right\}_{n=N_{0}}^{\infty}$ and arbitrarily chosen $N \times N$ matrices $H_{1}, \ldots, H_{N_{0}-1}$, such that for all $n \geq N_{0}$,

$$
\log \operatorname{det} T_{n}(a)=(n+1) \log G(a)+\operatorname{tr}\left(H_{1}+\cdots+H_{n}\right)+\log \widetilde{E}(a)+\sum_{k=n+1}^{\infty} s_{k},
$$

where the constant $G(a)$ is given by (2).

3.3. The key estimate. The following result will be used to show that a decomposition (19) satisfying all requirements of Lemma 3.2 exists.

Proposition 3.3. Suppose $\omega: \mathbb{Z} \rightarrow[1, \infty)$ is a weight satisfying (7)-(8). Let $\Sigma$ be a compact set in the complex plane and b,c: $\Sigma \rightarrow\left(W_{\omega}\right)_{N \times N}$ be continuous functions. For $n, k \in \mathbb{Z}_{+}$and $\lambda \in \Sigma$, put

$$
G_{n, k}(\lambda):=G_{n, k}(b(\lambda), c(\lambda)), \quad M_{n}(b, c):=\left(\max _{\lambda \in \Sigma} R_{n}^{+}(b(\lambda))\right)\left(\max _{\lambda \in \Sigma} R_{n}^{-}(c(\lambda))\right)
$$


where $G_{n, k}(\cdot, \cdot)$ and $R_{n}^{ \pm}(\cdot)$ are defined by (6) and (13), respectively. If $p \in \mathbb{N}$, then there exist a constant $C_{p} \in(0, \infty)$ and a number $N_{0} \in \mathbb{N}$ such that

$$
\begin{aligned}
& \left|\operatorname{tr} \log \left\{I-\sum_{k=0}^{\infty} G_{n, k}(\lambda)\right\}+\operatorname{tr}\left[\sum_{j=1}^{p-1} \frac{1}{j}\left(\sum_{k=0}^{p-j-1} G_{n, k}(\lambda)\right)^{j}\right]\right| \\
& \leq C_{p}\left[\varphi_{n} M_{n}(b, c)\right]^{p}
\end{aligned}
$$

for all $\lambda \in \Sigma$ and all $n \geq N_{0}$.

Proof. For $n, m \in \mathbb{Z}_{+}$and $\lambda \in \Sigma$, put

$$
A_{n}(m, \lambda):=\sum_{k=0}^{m-1} G_{n, k}(\lambda), B_{n}(m, \lambda):=\sum_{k=m}^{\infty} G_{n, k}(\lambda), C_{n}(m, \lambda):=\sum_{j=m}^{\infty} \frac{1}{j} B_{n}^{j}(0, \lambda) .
$$

Then

$$
\begin{aligned}
& \log \left\{I-\sum_{k=0}^{\infty} G_{n, k}(\lambda)\right\}+\sum_{j=1}^{p-1} \frac{1}{j}\left(\sum_{k=0}^{p-j-1} G_{n, k}(\lambda)\right)^{j} \\
& =-C_{n}(1, \lambda)+\sum_{j=1}^{p-1} \frac{1}{j} B_{n}^{j}(0, \lambda)-\sum_{j=1}^{p-1} \frac{1}{j} B_{n}^{j}(0, \lambda)+\sum_{j=1}^{p-1} \frac{1}{j} A_{n}^{j}(p-j, \lambda) \\
& =-C_{n}(p, \lambda)-\sum_{j=1}^{p-1} \frac{1}{j}\left[A_{n}(p-j, \lambda)+B_{n}(p-j, \lambda)\right]^{j}+\sum_{j=1}^{p-1} \frac{1}{j} A_{n}^{j}(p-j, \lambda) .
\end{aligned}
$$

Taking into account that

$$
\operatorname{tr} A_{n}^{\alpha}(m, \lambda) B_{n}^{\beta}(m, \lambda)=\operatorname{tr} B_{n}^{\beta}(m, \lambda) A_{n}^{\alpha}(m, \lambda)
$$

for all $\alpha, \beta \in \mathbb{N}$ and all $m \in \mathbb{Z}_{+}$, we get

$$
\begin{aligned}
\operatorname{tr}\left[\sum _ { j = 1 } ^ { p - 1 } \frac { 1 } { j } \left[A_{n}(p-j, \lambda)\right.\right. & \left.\left.+B_{n}(p-j, \lambda)\right]^{j}-\sum_{j=1}^{p-1} \frac{1}{j} A_{n}^{j}(p-j, \lambda)\right] \\
& =\sum_{j=1}^{p-1} \sum_{\ell=0}^{j-1}\left(\begin{array}{c}
j-1 \\
\ell
\end{array}\right) \operatorname{tr}\left[A_{n}^{\ell}(p-j, \lambda) B_{n}^{j-\ell}(p-j, \lambda)\right] .
\end{aligned}
$$

Let us estimate $\left\|A_{n}^{\ell}(p-j, \lambda)\right\|$ and $\left\|B_{n}^{j-\ell}(p-j, \lambda)\right\|$. In view of Proposition 2.3, taking into account (8), one can choose a number $N_{0} \in \mathbb{N}$ such that

$$
\varphi_{n} M_{n}(b, c)<1
$$


for all $n \geq N_{0}$. Then, by Proposition 2.4 and (23),

$$
\begin{aligned}
\left\|A_{n}^{\ell}(p-j, \lambda)\right\| & \leq\left\|A_{n}(p-j, \lambda)\right\|^{\ell} \\
& \leq\left(\sum_{k=0}^{p-j-1}\left\|G_{n, k}(\lambda)\right\|\right)^{\ell} \\
& \leq\left(\sum_{k=0}^{p-j-1}\left[\varphi_{n} M_{n}(b, c)\right]^{k+1}\right)^{\ell} \\
& \leq\left(\sum_{k=0}^{p-j-1} \max _{0 \leq k \leq p-1}\left[\varphi_{n} M_{n}(b, c)\right]^{k+1}\right)^{\ell} \\
& =\left[(p-j) \varphi_{n} M_{n}(b, c)\right]^{\ell} \\
& \leq p^{p}\left[\varphi_{n} M_{n}(b, c)\right]^{\ell}
\end{aligned}
$$

for all $j \in\{1, \ldots, p-1\}, \ell \in\{0, \ldots, j-1\}, n \geq N_{0}$, and $\lambda \in \Sigma$.

Similarly, taking into account Proposition 2.4 and (23), we obtain

$$
\begin{aligned}
\left\|B_{n}^{j-\ell}(p-j, \lambda)\right\| & \leq\left\|B_{n}(p-j, \lambda)\right\|^{j-\ell} \\
& \leq\left(\sum_{k=p-j}^{\infty}\left\|G_{n, k}(\lambda)\right\|\right)^{j-\ell} \\
& \leq\left(\sum_{k=p-j}^{\infty}\left[\varphi_{n} M_{n}(b, c)\right]^{k+1}\right)^{j-\ell} \\
& =\left(\frac{\left[\varphi_{n} M_{n}(b, c)\right]^{p-j+1}}{1-\varphi_{n} M_{n}(b, c)}\right)^{j-\ell} \\
& \leq\left[\varphi_{n} M_{n}(b, c)\right]^{(p-j+1)(j-\ell)}
\end{aligned}
$$

for all $j \in\{1, \ldots, p-1\}, \ell \in\{0, \ldots, j-1\}, n \geq N_{0}$, and $\lambda \in \Sigma$.

It is easy to see that

$$
(p-j+1)(j-\ell)+\ell-p=(p-j)(j-1-\ell) .
$$

Since $j \in\{1, \ldots, p-1\}$ and $\ell \in\{0, \ldots, j-1\}$, the latter equality implies that

$$
(p-j+1)(j-\ell)+\ell \geq p .
$$

From (23)-(26) we get for $j \in\{1, \ldots, p-1\}, \ell \in\{0, \ldots, j-1\}, n \geq N_{0}$, and $\lambda \in \Sigma$,

$$
\begin{aligned}
\left|\operatorname{tr}\left[A_{n}^{\ell}(p-j, \lambda) B_{n}^{j-\ell}(p-j, \lambda)\right]\right| & \leq\left\|A_{n}^{\ell}(p-j, \lambda) B_{n}^{j-\ell}(p-j, \lambda)\right\| \\
& \leq p^{p}\left[\varphi_{n} M_{n}(b, c)\right]^{\ell}\left[\varphi_{n} M_{n}(b, c)\right]^{(p-j+1)(j-\ell)} \\
& \leq p^{p}\left[\varphi_{n} M_{n}(b, c)\right]^{p}
\end{aligned}
$$


Combining (22) and (27), we obtain

$$
\begin{aligned}
\operatorname{tr}\left[\sum_{j=1}^{p-1} \frac{1}{j}\left[A_{n}(p-j, \lambda)+B_{n}(p-j, \lambda)\right]^{j}-\sum_{j=1}^{p-1} \frac{1}{j} A_{n}^{j}(p-j, \lambda)\right] \mid \\
\leq \sum_{j=1}^{p-1} \sum_{\ell=0}^{j-1}\left(\begin{array}{c}
j-1 \\
\ell
\end{array}\right) p^{p}\left[\varphi_{n} M_{n}(b, c)\right]^{p} \\
=\widetilde{C}_{p}\left[\varphi_{n} M_{n}(b, c)\right]^{p}
\end{aligned}
$$

where $\widetilde{C}_{p}:=p^{p} \sum_{j=1}^{p-1} 2^{j-1}$. Similarly we can estimate the trace of $C_{n}(p, \lambda)$ :

$$
\begin{aligned}
\left|\operatorname{tr} C_{n}(p, \lambda)\right| & \leq \sum_{j=p}^{\infty} \frac{1}{j}\left\|\sum_{k=0}^{\infty} G_{n, k}(\lambda)\right\|^{j} \\
& \leq \sum_{j=p}^{\infty}\left(\sum_{k=0}^{\infty}\left\|G_{n, k}(\lambda)\right\|\right)^{j} \\
& \leq \sum_{j=p}^{\infty}\left(\sum_{k=0}^{\infty}\left[\varphi_{n} M_{n}(b, c)\right]^{k+1}\right)^{j} \\
& =\sum_{j=p}^{\infty}\left(\frac{\varphi_{n} M_{n}(b, c)}{1-\varphi_{n} M_{n}(b, c)}\right)^{j} \\
& \leq \sum_{j=p}^{\infty}\left[\varphi_{n} M_{n}(b, c)\right]^{j} \\
& =\frac{\left[\varphi_{n} M_{n}(b, c)\right]^{p}}{1-\varphi_{n} M_{n}(b, c)} \\
& \leq\left[\varphi_{n} M_{n}(b, c)\right]^{p}
\end{aligned}
$$

for all $\lambda \in \Sigma$ and all $n \geq N_{0}$. Combining (21) and (28)-(29), we arrive at (20) with $C_{p}=1+\widetilde{C}_{p}$.

3.4. Proof of Theorem 1.4. Part (a) follows from Proposition 2.2 and Theorem 2.1 .

Let $p \in \mathbb{N}$ and $b, c: \Sigma \rightarrow\left(W_{\omega}\right)_{N \times N}$ be chosen as in part (a). By Proposition 3.3, there exists a number $N_{0} \in \mathbb{N}$ such that for all $n \geq N_{0}$ and all $\lambda \in \Sigma$,

$$
\begin{aligned}
\operatorname{tr} \log \left\{I-\sum_{k=0}^{\infty} G_{n, k}(\lambda)\right\}= & -\operatorname{tr}\left[\sum_{j=1}^{p-1} \frac{1}{j}\left(\sum_{k=0}^{p-j-1} G_{n, k}(\lambda)\right)^{j}\right] \\
& +O\left(\left[\varphi_{n} M_{n}(b, c)\right]^{p}\right)
\end{aligned}
$$


and $O\left(\left[\varphi_{n} M_{n}(b, c)\right]^{p}\right)$ is uniform with respect to $\lambda \in \Sigma$ and $n \geq N_{0}$. Obviously,

$$
M_{k+1}(b, c) \leq M_{k}(b, c) \quad \text { for all } k \in \mathbb{Z}_{+} .
$$

From (31) and Proposition 2.3 it follows that

$$
\sum_{k=n+1}^{\infty}\left[\varphi_{k} M_{k}(b, c)\right]^{p} \leq\left[M_{n}(b, c)\right]^{p} \sum_{k=n+1}^{\infty} \varphi_{k}^{p}=o\left(\sum_{k=n+1}^{\infty} \varphi_{k}^{p}\right) \quad \text { as } n \rightarrow \infty
$$

and this holds uniformly with respect to $\lambda \in \Sigma$.

Applying Lemma 3.2 to the decomposition(30) and taking into account (32), we deduce that for every $\lambda \in \Sigma$ there exists a constant $\widetilde{E}(a, \lambda) \neq 0$ such that (11) is satisfied for every $p \in \mathbb{N}$ uniformly with respect to $\lambda \in \Sigma$. This finishes the proof of part (c).

If we take $p=1$ in (11), then

$$
\log \operatorname{det} T_{n}(a(\lambda))=(n+1) \log G(a(\lambda))+\log \widetilde{E}(a, \lambda)+o\left(\sum_{k=n+1}^{\infty} \varphi_{k}\right)
$$

and the convergence is uniform with respect to $\lambda \in \Sigma$.

On the other hand, by Theorem 3.1, $T(a(\lambda)) T\left([a(\lambda)]^{-1}\right)-I$ is of trace class and

$$
\log \operatorname{det} T_{n}(a(\lambda))=(n+1) \log G(a(\lambda))+\log \operatorname{det} T(a(\lambda)) T\left([a(\lambda)]^{-1}\right)+o(1)
$$

as $n \rightarrow \infty$ for every $\lambda \in \Sigma$. Combining (33) and (34), we get

$$
\log \widetilde{E}(a, \lambda)=\log \operatorname{det} T(a(\lambda)) T\left([a(\lambda)]^{-1}\right)
$$

and therefore (10) holds uniformly with respect to $\lambda \in \Sigma$. Part (b) and Theorem 1.4 are proved.

3.5. Proof of Theorem 1.5. Suppose $\lambda \notin \operatorname{sp} T(a) \cup \operatorname{sp} T(\widetilde{a})$. Since $a-\lambda$ is continuous with respect to $\lambda$ as a function from a neighborhood of $\partial \Omega$ to $\left(W_{\omega}\right)_{N \times N}$, Theorem 1.4(b) shows that

$$
\begin{aligned}
\log \operatorname{det} T_{n}(a-\lambda)= & (n+1) \log G(a-\lambda)+\log \operatorname{det} T[a-\lambda] T\left[(a-\lambda)^{-1}\right] \\
& +o\left(\sum_{k=n+1}^{\infty} \varphi_{k}\right) \quad \text { as } n \rightarrow \infty
\end{aligned}
$$

and the convergence is uniform with respect to $\lambda$ in a neighborhood of $\partial \Omega$. Hence, we can differentiate both sides with respect to $\lambda$, multiply by $f(\lambda)$, and integrate over $\partial \Omega$. The proof is finished by a literal repetition of Widom's proof of Theorem 1.2 (see [16, p. 21] or [4, Section 10.90]) with $o(1)$ replaced by the same $o(\ldots)$ as in the above formula. 
Acknowledgements. This work is partially supported by the grant of F.C.T. (Portugal) FCT/FEDER/POCTI/MAT/59972/2004.

\section{References}

[1] Böttcher, A. and Silbermann, B., Notes on the asymptotic behavior of block Toeplitz matrices and determinants. Math. Nachr. 98 (1980), 183 - 210.

[2] Böttcher, A. and Silbermann, B., Invertibility and Asymptotics of Toeplitz Matrices. Berlin: Akademie-Verlag 1983.

[3] Böttcher, A. and Silbermann, B., Introduction to Large Truncated Toeplitz Matrices. New York: Springer 1999.

[4] Böttcher, A. and Silbermann, B., Analysis of Toeplitz Operators (2nd edition). Berlin: Springer 2006.

[5] Ehrhardt, T., A new algebraic approach to the Szegö-Widom limit theorem. Acta Math. Hungar. 99 (2003), 233 - 261.

[6] Gelfand, I., Raikov, D. and Shilov, G., Commutative Normed Rings. New York: Chelsea 1964.

[7] Gohberg, I., Goldberg, S. and Kaashoek, M., Classes of Linear Operators, Vol. II. Basel: Birkhäuser 1993.

[8] Gohberg, I. and Krein, M. G., Introduction to the Theory of Linear Nonselfadjoint Operators. Providence (RI): American Mathematical Society 1969.

[9] Grenander, U. and Szegö, G., Toeplitz Forms and their Applications. Berkeley (LA): University of California Press 1958.

[10] Karlovich, A., Higher order asymptotics of Toeplitz determinants with symbols in weighted Wiener algebras. J. Math. Anal. Appl. 320 (2006), 944 - 963.

[11] Krein, M. G., Certain new Banach algebras and theorems of the type of the Wiener-Lévy theorems for series and Fourier integrals (in Russian). Mat. Issled. 1 (1966)(1), $82-109$.

[12] Litvinchuk, G. S. and Spitkovsky, I. M., Factorization of Measurable Matrix Functions. Basel: Birkhäuser 1987.

[13] Shubin, M. A., Factorization of matrix functions depending on a parameter in normed rings and related problems of the theory of Noetherian operators (in Russian). Matem. Sbornik 73(115) (1967), 610 - 629.

[14] Simon, B., Orthogonal Polynomials on the Unit Circle, Part 1. Providence (RI): American Mathematical Society 2005.

[15] Vasil'ev, V. A., Maksimenko, E. A. and Simonenko, I. B., On a Szegö-Widom limit theorem (in Russian). Dokl. Akad. Nauk 393 (2003), $307-308$.

[16] Widom, H., Asymptotic behavior of block Toeplitz matrices and determinants. II. Advances in Math. 21 (1976), 1 - 29.

Received September 16, 2005 